\title{
Formación inicial docente: preocupación de la educadora Beatrice Ávalos, Premio Nacional de Educación 2013, Chile
}

\author{
Jaime Caiceo Escudero \\ Universidad de Santiago de Chile - USACH, Chile \\ Apoio: Universidad de Santiago de Chile, \\ Departamento de Educación
}

\section{RESUMO}

A qualidade da educação é uma das maiores preocupações no Chile desde a volta da democracia em 1990. Em efeito, nessa época criou-se o Programa de Melhoramento da Qualidade e Equidade da Educação - MECE, dependendo do Ministério da Educação, com tal objetivo. Nesta comunicação se persegue dar a conhecer o pensamento e a ação pedagógica da Dra. Ávalos com o fim de compreender os distintos programas implementados no Chile nos últimos 25 anos, em vistas de melhorar a qualidade da educação, enfatizando sua preocupação pela formação inicial, aspecto chave que teve presente na Comissão que lhe concedeu o Prêmio Nacional em Ciências da Educação no ano 2013. Ao mesmo tempo, se reconstruirá sua biografia e o trabalho acadêmico realizado durante seu autoexílio em Gales, Canadá e Papua Guine-a, sempre na linha de melhorar a qualidade docente.

PALAVRAS-CHAVE: Qualidade da Educação. Formação Inicial Docente. Pensamento Educativo. Biografia do Prêmio Nacional.

\section{INITIAL TEACHER TRAINING: CONCERN OF THE EDUCATOR BEATRICE AVALOS, NATIONAL EDUCATION PRIZE 2013, CHILE}

\begin{abstract}
The quality of education is one of the biggest concerns in Chile since the return to democracy in 1990. In fact, at that time, it was created the Improvement Quality and Equity Educational program - MECE, which had an objective dependent on the Chilean Ministry of Education. This paper is intended to inform Dr. Avalos thinking and pedagogical action in order to understand the different programs implemented in Chile in the last 25 years, with the goal of improving the quality of education emphasizing her concern on the initial training, which was a key aspect that the Commission considered to grant her the Educational Sciences National Prize in 2013. At the same time, it will be rebuild her biography and academic work performed during her self-imposed exile in Canada, Wales and Papua Guinea, always in the line of improving teacher's quality.
\end{abstract}

KEYWORDS: Quality of Education. Initial Teacher Training. Educational Thinking. National Prize Biography.

FORMACIÓN INICIAL DOCENTE: PREOCUPACIÓN DE LA EDUCADORA BEATRICE ÁVALOS, PREMIO NACIONAL DE EDUCACIÓN 2013, CHILE 


\section{RESUMEN}

La calidad de la educación es una de las mayores preocupaciones en Chile desde la vuelta a la democracia en 1990. En efecto, en esa época se creó el Programa de Mejoramiento de la Calidad y Equidad de la Educación - MECE, dependiente del Ministerio de Educación, con tal objetivo. En esta comunicación se persigue dar a conocer el pensamiento y la acción pedagógica de la Dra. Ávalos con el fin de comprender los distintos programas implementados en Chile en los últimos 25 años, en vista de mejorar la calidad de la educación, enfatizando su preocupación por la formación inicial, aspecto clave que tuvo presente la Comisión que le otorgó el Premio Nacional en Ciencias de la Educación en el año 2013. Al mismo tiempo, se reconstruirá su biografía y el trabajo académico realizado durante su autoexilio en Gales, Canadá y Papúa Guinea, siempre en la línea de mejorar la calidad docente.

PALABRAS CLAVE: Calidad de la Educación. Formación Inicial Docente. Pensamiento Educativo. Biografía Premio Nacional.

\section{INTRODUCCIÓN}

La Dra. Beatrice Ávalos estuvo 20 años fuera del país (1974-1994) y al volver, el Ministro de Educación de la época, Ernesto Schiefelbein, la nombra como profesional integrante del Programa MECE -Media, iniciado en el primer gobierno democrático después de la dictadura, y en esa condición crea y coordina el Programa de Desarrollo Profesional Colaborativo para profesores de Educación Media, al cual se le llamó GPT (Grupos Profesionales de Trabajo), pues de acuerdo a su pensamiento, el trabajo en equipo de los docentes mejora el desempeño de los mismos en su quehacer, y se ven favorecidos sus alumnos. En el mismo Ministerio entre 1997 y el 2002 establece y coordina el Programa FFID (Fortalecimiento de la Formación Inicial Docente) con el objeto de mejorar la formación de los docentes en 17 universidades chilenas, tanto públicas como privadas; es en este aspecto que mayormente se ha destacado la Dra. Beatrice Ávalos y la hizo meritoria para recibir el Premio Nacional en Ciencias de la Educación en el 2013. Posteriormente, (2006-2010) coordinó la aplicación nacional de la prueba internacional, organizada por IEA y denominada TEDS-M , que perseguía examinar el estado de la formación docente en matemáticas de los futuros profesores de educación básica en el país; esta prueba ha sido el primer estudio comparativo del conocimiento en matemáticas de los docentes en su formación inicial.

La experiencia adquirida en el Ministerio de Educación, unida a su experiencia internacional, la lleva a establecerse en el Centro de Investigación Avanzado en Educación de la Universidad de Chile y proseguir con su vocación más específica: la investigación; llega a esta institución para dirigir inicialmente el Núcleo Milenio sobre la Profesión Docente en Chile en el año 2010. Va a desarrollar preferentemente investigaciones ligadas a la formación inicial 
docente y al trabajo colaborativo entre profesores novatos y experimentados. De esta forma, prosigue el tipo de trabajo que más le satisface académicamente y que había iniciado en 1972 en la Universidad Católica de Chile al crear, junto a Ernesto Schiefelbein, el Programa Interdisciplinario de Investigaciones en Educación - PIIE, que el Rector Delegado nombrado por la dictadura militar, Almirante Jorge Swett, lo elimina y es acogido por la Iglesia Católica, a través del Cardenal Raúl Silva Henríquez, en la Academia de Humanismo Cristiano, que crea para recibir a los académicos exonerados de las universidades durante el inicio de la dictadura (1973 en adelante). El PIIE llegará a convertirse en uno de los centros esenciales de generación de conocimiento en ciencias de la educación en Chile durante el oscuro período que se vivió entre 1973 y 1990, y es una de las contribuciones más destacadas de la Dra. Ávalos. Ese Programa aún subsiste.

En este artículo se persigue dar a conocer el pensamiento y la acción pedagógica de la Dra. Ávalos con el fin de comprender los distintos programas implementados en Chile en los últimos 25 años, en vista de mejorar la calidad de la educación, enfatizando su preocupación por la formación inicial, aspecto clave que tuvo presente la Comisión que le otorgó el Premio Nacional en Ciencias de la Educación en el año 2013. Al mismo tiempo, se reconstruirá su biografía y el trabajo académico realizado durante su autoexilio en Gales, Canadá y Papúa Guinea, siempre en la línea de mejorar la calidad docente.

Este trabajo se ubica en el paradigma cualitativo, propio de las ciencias sociales, con una epistemología fenomenológica y hermenéutica, es decir, se describirá y analizará interpretativamente la bibliografía examinada en búsqueda de lograr que el desarrollo del mismo sea veraz (CAICEO, 2018) y, a su vez, la metodología aplicada es la propia de los estudios históricos, recurriendo a fuentes primarias y secundarias, tales como análisis documental de los escritos de la Premio Nacional, entrevistas a actores directos y análisis de los programas realizados.

\section{PRINCIPALES RASGOS BIOGRÁFICOS Y FORMACIÓN DE BEATRICE ÁVALOS}

El mundo estaba recuperándose de la gran depresión que subsistió entre 1929 y 1932 que también afectó enormemente a Chile por el derrumbe de las exportaciones de salitre y cobre- y por la crisis política que se dio en el país entre 1925 y 1932 con la seguidilla de mandatarios, renuncias y ruido de sables, producto de las consecuencias de la debacle económica: Emiliano Figueroa Larraín (1925-1927), Carlos Ibáñez del Campo (1927-1931), Manuel Trucco (1931), Juan Esteban Montero (1931-1932), la República Socialista con 
Eugenio Matte, Marmaduke Grove y Carlos Dávila (junio a octubre de 1932) y, finalmente, la elección por segunda vez de Arturo Alessandri Palma (1932-1938) (BIBLIOTECA DEL CONGREESO NACIONAL, 1925-1973). En ese contexto histórico, nació Beatrice Ávalos Davidson en Santiago de Chile el 26 de febrero 1935, hija mayor de Luis Alejandro Ávalos Zamorano, de profesión ingeniero y de Elsie Davidson Wright, sin estudios superiores, pero trabajó como profesora de inglés en el mineral de Sewell y, antes, en el Instituto Chileno Británico de Cultura, ya que era de nacionalidad inglesa, nacida en Londres; el abuelo materno de Beatrice era escocés. Nuestra educadora tuvo dos hermanos: Eleanor, nacida el 21 enero 1938 - quien ha sido pianista - y Alejandro Juan, nacido el 16 de diciembre 1945, profesor de inglés, titulado en la Pontificia Universidad Católica de Chile (ÁVALOS, 2017, p. 1). El joven Alejandro es un desaparecido de la dictadura militar chilena (1973-1990) desde 1975; él fue detenido por la policía política del régimen en la misma universidad; allí trabajaba en el PIIE Programa fundado por su hermana Beatrice- en un Proyecto de Investigación con Ernesto Schiefelbein y, hasta esta fecha, no aparecen sus restos. En 1992 se realizó un funeral creyendo que unas osamentas encontradas en la zona militar de Peldehue, a $30 \mathrm{kms}$. de la capital, eran de Alejandro; sin embargo, lamentablemente en septiembre del año 2013, por una prueba de ADN, se concluyó que dichas osamentas no eran suyas. Beatrice, transitoriamente estaba en Chile cuando desapareció su hermano; gracias a ello, ayudó a calmar a su madre e iniciar la búsqueda, la cual fue infructuosa; ella se encontraba en ese año, invitada como profesora visitante en Gales. La desaparición de su hermano fue "la razón de quedarme en Gales, fue la recomendación de la embajada Británica dada la desaparición de Alejandro" (ÁVALOS, 2017, p. 2). En otro lugar, complementa: "Estuve dos años allá y decidí quedarme. No me gustaba lo que estaba ocurriendo en Chile en esa época y no tenía ganas de regresar" (ÁVALOS, 2014a, p. 43. Respecto al impacto que la desaparición de Alejandro significó, acota: "Fue un tremendo golpe para la familia y eso me hizo postergar mi vuelta a Chile. Ese año tenía que haber regresado (1975), pero por consejo recibidos no lo hice. Además, gané un concurso para ocupar un puesto como profesora titular en Gales y durante una década seguí mi vida académica en Inglaterra" (Ibid, p. 43).

El pesar que este acontecimiento le provocó a Beatrice fue mayor, en consideración a que ella, en Santiago, vivía en el centro de la ciudad con su hermano en la calle Almirante Barroso, antes de viajar a Gales (ÁVALOS, 2013). Sin embargo, 38 años después de la desaparición de su hermano tuvo una gran alegría, puesto que el 5 de septiembre de 2013 recibió de la Pontificia Universidad Católica de Chile el título de Profesor de Inglés de Alejandro 
Ávalos en forma póstuma, gracias a las gestiones que realizó el Rector de esa universidad, Ignacio Sánchez.

Beatrice Ávalos realizó sus estudios primarios y secundarios en un establecimiento público, el Liceo de Niñas $\mathrm{N}^{\circ}$ 7, de la comuna de Providencia, en la Región Metropolitana entre 1941 y 1952. Al año siguiente, ingresó a la Escuela de Pedagogía de la Pontificia Universidad Católica de Chile a la carrera de Historia y Geografía, y obtuvo su título de Profesora en Historia y Geografía en 1958. Empero, su proyecto de vida lo proyectaba en el ámbito del derecho, a lo cual, su madre se opuso. Como hablaba inglés correctamente, por la formación materna, pensó que para tener buenos ingresos -las secretarias ganaban más que los profesores- podía estudiar paralelamente, por las mañanas, Secretariado Bilingüe; para ello ingresó al Instituto Británico que, en ese entonces se le conocía como New Morning School, en "donde aprendíamos taquigrafía, dactilografía, esas cosas que hoy día ni se enseñan" (ÁVALOS, 2014b, p. 1). Sus actividades universitarias eran por las tardes.

Mientras realizaba sus estudios universitarios, ingresó en 1956 a las Hermanas Marianas de Schöenstatt “porque me pareció una institución interesante en su carácter de Instituto Secular desde la cual podría tener una acción desde la perspectiva católica que tuviese incidencia social (en el sentido amplio de la palabra)" (ÁVALOS, 2017, p. 2).

Gracias a que obtuvo una beca Fulbright, pudo efectuar estudios de doctorado en St. Louis University, Estados Unidos, entre 1959 y 1961; en esa universidad alcanzó el grado de Ph.D. con concentración mayor en Educación y menor en Historia y Filosofía en julio de 1961 (IBID). El haber conseguido esta beca fue algo excepcional, pues la mayoría de las becas eran entregadas en ese tiempo a hombres, pero la calidad académica y la personalidad de Beatrice hicieron posible que ella la obtuviera. Su tesis doctoral se denominó New Men for New Times ; en ella realiza un análisis de la educación, desde un punto de vista filosófico-educacional, desde tres posiciones diferentes: Dewey, Marx y el cristianismo; basado en ello plantea su posición respecto a cómo debe educarse el hombre nuevo para los nuevos tiempos, situación existencial del hombre de la segunda mitad del siglo pasado.

En relación a su beca y a sus estudios en Norteamérica, la educadora comenta:

Pero lo de la beca fue raro porque era la primera vez que me subía a un avión. En ese tiempo te sacaban fotos cuando te subías a este medio de transporte, para la vida social o no sé qué. Era un gran evento irse en avión a Estados Unidos. Había pocas becas en Chile, y esa beca Fullbright estaba recién empezando, no había otra fuente de becas (ÁVALOS, 2014b, p. 2). 
Esta permanencia en el país del norte fue muy significativa porque en ese entonces no vislumbraba que fuera el preámbulo de tantos viajes que posteriormente ha realizado por diferentes continentes, investigando temas educativos y perfeccionando o formando profesores.

Respecto a su vocación de educadora, señala:

Siempre me preguntan si cuando chica ya quería dedicarme a ser profesora y a la formación. Pero a todos les contesto que de chica ni se me pasó por la mente que iba a dedicarme a esto. Aunque, recuerdo que cuando teníamos vacaciones de invierno, con los vecinos de la casa de al lado nos juntábamos y hacíamos nuestra propia sala de clases. Cada uno le enseñaba una materia al otro. Quizás ahí partió todo (ÁVALOS, 2014b, p. 3).

En relación a su personalidad, uno de sus ayudantes en la Universidad Católica sostiene que es "una educadora muy rigurosa, inteligente, con sólida formación y una persona con gran calidad humana" (BURROWS, 2014, p. 2). El mismo estudiante en cuanto a su beca y el haber ido muy joven a estudiar a Estados Unidos indica: "fue una señal clara de su capacidad y decisión de avanzar en el conocimiento pedagógico" (p. 2).

Otro exalumno y, posteriormente, colega de investigación en el PIIE, acota sobre ella:

Sin duda tiene características de líder, de personalidad fuerte. Beatrice es como locomotora, pero, además, es como muy sensitiva, muy asequible para mí, porque a lo mejor tengo una relación más personal con ella porque la conocí siendo el primer alumno que se graduó con ella en el Magíster en la Universidad Católica. Pensábamos más o menos igual frente a la universidad. Yo tengo una relación con ella de mucho cariño, de mucha admiración, pero no puedo dejar de decir que ella tiene una personalidad fuerte [...]. Pero si ella piensa algo, defiende eso que piensa con dientes, muelas, uñas, sables, todo [...]. Es muy consistente, muy coherente en todo lo que ella hace, una persona muy sólida en lo que piensa, muy de una línea, muy confiable, muy responsable de su función pedagógica" (GONZÁLEZ, 2014, p. 2).

\section{ACCIÓN PEDAGÓGICA DE LA EDUCADORA BEATRICE ÁVALOS}

\subsection{Docencia en la educación secundaria}

Una vez que regresó a Chile con su doctorado, Beatrice Ávalos se integró como profesora de Historia y Geografía al Colegio Mariano, perteneciente a su institución religiosa; allí ejerció la docencia en la educación secundaria entre 1962 y 1968. De esta forma conoció directamente el sistema escolar chileno, con sus aciertos y fracasos. Esto le permitió poder, junto al P. Patricio Cariola, s.j. - Presidente de la FIDE - (CAICEO, 2012a), realizar un aporte a la Reforma Educacional iniciada en 1965 durante el gobierno de Eduardo Frei Montalva, la 
cual se inspiraba en lo filosófico en el humanismo cristiano y en lo pedagógico en los planteamientos renovados de Tyler y Bloom (CAICEO, 2016).

\subsection{Su vida académica en la Pontificia Universidad Católica de Chile}

En forma paralela, regresó a su Casa de Estudios, la Pontificia Universidad Católica de Chile, como académica en la Escuela de Pedagogía, para dictar las cátedras de Pedagogía General y de Filosofía de la Educación, entre 1962 y 1974; al mismo tiempo, ejerció el cargo de Subdirectora de la misma Escuela entre 1968 y 1970 (ÁVALOS, 2015a). Allí le tocó vivir una experiencia significativa, a saber, participar activamente en la Reforma Universitaria iniciada en esa universidad el 11 de agosto de 1967, gracias a lo cual, por el espíritu democratizador del proceso, fue elegida representante de los académicos en el Consejo Superior de la universidad en 1968, función que ejerció hasta 1971. A su vez, por su compromiso con la reforma, el Rector Fernando Castillo, la designó Coordinadora de las Sedes Regionales de la Universidad en 1970 (Talca, Concepción, Temuco y Villarrica), cargo que mantuvo hasta el golpe miliar del 11 de septiembre de 1973; un mes después, la universidad fue intervenida por un Rector Designado por la dictadura militar, el Almirante Jorge Swett. En relación al proceso de reforma, acota: "Estuve junto a los estudiantes y a un grupo de profesores de la PUC porque estábamos convencidos de la necesidad de reforma. Participé en la reestructuración posterior de la universidad y fui miembro del Consejo Superior" (ÁVALOS, 2017, p. 3).

Sin embargo, su acción más significativa desde el punto de vista académico en esta universidad fue haber participado en la creación de dos programas muy importantes y que aún subsisten; ellos son el PIIE y el Programa de Magíster en Educación. El PIIE perseguía que la universidad tuviera una instancia de investigación educativa, a fin de cumplir con una de las funciones básicas que la universidad reformada debía tener como uno de sus ejes centrales: la investigación con el objeto de entregar docencia actualizada y de calidad. Para lograr su creación en 1971 tuvo la activa participación de los académicos Ernesto Schiefelbein, chileno, Premio Nacional de Educación 2007 y de Noel McGinn, norteamericano, profesor de Harvard. En este Programa, dependiente de la Vicerrectoría Académica, Beatrice Ávalos se desempeñó como Subdirectora entre 1971 hasta 1974, fecha en que ella se fue a Gales.

En cuanto al Programa de Magíster en Educación, lo creó en 1972, dependiente de la Facultad de Educación de la Universidad Católica, desempeñando el cargo de Jefa del Programa; con esta creación demuestra el interés que tenía por reforzar la educación inicial en 
la formación docente. Este Programa aún subsiste y fue la base, después de varios años, del Doctorado que creó la misma Facultad.

Con el objeto de relacionar a las dos entidades mencionadas con universidades extranjeras, Beatrice Ávalos se contactó con la Universidad de Gales y fue a esa entidad en 1974. Al respecto, un colega comenta la relación con la universidad británica: "Después de eso ella se fue a Gales, invitada porque dentro de este programa del PIIE había un convenio con la Universidad de Gales y vinieron profesores galeses, no solo británicos, a trabajar con nosotros en investigación o en programas de magíster" (GONZÁLEZ, 2014, p. 2).

El mismo año abandona la institución religiosa a la que pertenecía porque, en sus propias palabras, "me retiré cuando me di cuenta de que había una tensión entre el trabajo profesional y social que ejercía y el foco efectivo del Instituto" (ÁVALOS, 2017, p. 2).

\subsection{Su autoexilio (1974-1994)}

Instalada en Gales (1974), Beatrice Ávalos, conoció a su futuro marido, quien fue profesor y Rector de la Universidad de Gales. Contrajo matrimonio en "1985 en Cardiff, País de Gales con Cecil Wilfrid Bevan" (IBID, p. 3); "era un tipo encantador, muy agradable" (GONZÁLEZ, 2014, p. 4); en Inglaterra para mantener su apellido, al momento de casarse, se puso Ávalos-Bevan, ya que las mujeres inglesas adoptan el apellido del marido (ÁVALOS, 2013: s/p). Su permanencia en ese país como académica -ejerciendo como docente y en cargos administrativo-académicos- duró hasta fines de los 80', época en la que la situación se empezó a poner difícil por las restricciones económicas impuestas a las universidades por Margaret Thatcher. Al respecto, la académica señala: "Ella empieza a hacerle la vida imposible a las universidades. En la que yo estaba trabajando, por ejemplo, ordena el traslado de la Facultad de Educación. Mi marido estaba jubilado, no tenía más que hacer y yo no estaba comprometida con el nuevo proyecto de la universidad" (ÁVALOS, 2013: s/p).

Mientras estuvo en Gales fue Profesora Visitante en Canadá en tres entidades superiores: University of New Brunswick, Universidad de Toronto y en el IDRC ; ella misma describe lo más importante que allí realizó: en las universidades fue académica y en la tercera institución contribuyó “a interesar al IDRC para que prestara apoyo a los centros de investigación en Ciencias Sociales en los países con régimen de dictadura en América Latina, incluyendo el PIIE y CIEPLAN en Chile” (ÁVALOS, 2015b, p. 5).

Por la situación compleja que vivía en la Universidad de Gales postuló a un cargo como académica a un país de habla inglesa en vías de desarrollo. Al respecto, señala: 
[...] Así concurso y gano la cátedra de Educación de la Universidad de Papúa Nueva Guinea (PNG), donde permanecí entre 1988 y 1994. Durante ese tiempo me desempeñé como Jefe del Departamento de Educación, establecí el primer Programa de Magíster en Educación de la Universidad de PNG y establecí convenios con el Queensland University of Technology (Australia) para la preparación conjunta de Bachilleres en Educación en la Universidad de PNG (ÁVALOS, 2015b, p. 6).

Mientras se encontraba en la polinesia murió su esposo de una embolia, cuando había ido a Inglaterra y ella estaba en Papúa Nueva Guinea. A pesar del dolor que aquello le produjo, Beatrice Ávalos permaneció en ese lugar elegido porque su preocupación era ayudar a mejorar la educación y, especialmente, la formación inicial; por ello creó el Magíster en Educación.

\section{SU ACCIÓN PEDAGÓGICA EN CHILE A PARTIR DE 1994}

\subsection{Su trabajo en el Ministerio de Educación}

Con el regreso a la democracia en Chile en 1990, Beatrice Ávalos visitaba más a menudo su país y, de esta forma, en 1994 sucedió lo que ella misma relata:

Yo había venido antes a mi país y Cristián Cox me preguntó si quería volver y hacerme cargo como Coordinadora del Programa Mejoramiento de la Calidad y Equidad de la Educación - MECE. Acepté y me quedé en el Ministerio de Educación hasta 2008. Pero luego, la Universidad de Chile me pidió que postulara a un proyecto grande en el Centro de Investigación Avanzada en Educación -CIAE (ÁVALOS, 2013, s/p).

En el Ministerio de Educación desempeñó varias funciones, que ella misma describe:

Como integrante del programa MECE-Media del Ministerio de Educación creo y coordino un programa de desarrollo profesional colaborativo para profesores de Educación Media conocido como los Grupos Profesionales de Trabajo (GPT), y entre 1997-2002 establezco y coordino el Programa de Fortalecimiento de la Formación Inicial Docente (FFID) que contribuyó a mejorar la formación docente en 17 universidades públicas y privadas. Más tarde, coordino la aplicación nacional de la prueba internacional IEA TEDSM que examinó el estado de la formación docente en matemáticas de los futuros profesores de educación básica (2006-2010) (ÁVALOS, 2015b, s/p).

De la labor realizada, la que más le satisfizo fue el Programa de Fortalecimiento de la Educación Inicial, pues allí es donde se logra cambiar la visión de la carrera de profesor; se comienza a valorar más porque los docentes se vieron fortalecidos en su labor y, por lo mismo, hubo más postulantes a la carrera de pedagogía. En relación a la formación inicial, ella indica: 
Si yo tuviera el poder, armaría un Centro dependiente del Ministerio de Educación que fuera de coordinación de la formación docente, de apoyo, de monitoreo, de investigación en torno a la formación docente. Lo haría en el Ministerio de Educación porque creo que es esa institución la que tiene la responsabilidad de hacer esto y esa es la entidad que tendría excelentes académicos y profesionales que trabajarían en vinculación con ese Centro (ÁVALOS, 2014b, p. 6).

\subsection{Su trabajo como investigadora en el CIAE}

El Centro de Investigación Avanzado en Educación de la Universidad de Chile la llamó en el 2008 para que fuera la Investigadora Responsable del Núcleo Milenio: La Profesión Docente en Chile, Políticas, Prácticas, Proyecciones; esta investigación duró hasta el 2011. Desde entonces, se mantiene hasta la actualidad como Investigadora Asociada en esa institución, desarrollando diversos estudios como el de seguimiento de profesores de la universidad en el ejercicio profesional. Sus áreas de interés en investigación son: Formación de profesores, educación y desarrollo, prácticas pedagógicas, las prácticas de aula, la mujer en el desarrollo social y políticas educacionales en países en desarrollo (ÁVALOS, 2015b).

Su trabajo académico como investigadora no la ha apartado de la docencia; ella la ejerce en los doctorados de la Facultad de Educación de la Pontificia Universidad Católica, de la Universidad de Playa Ancha en Valparaíso y en la Universidad ORT de Uruguay; sus áreas de docencia preferidas son: Introducción a la Teoría Educacional, Filosofía e Historia de la Educación, Metodología de la Investigación Educacional (especialmente los enfoques cualitativos) y Políticas y Educación. A su vez, ha sido Presidenta de las Juntas Directivas de la Universidad Metropolitana de Ciencias de la Educación y del PIIE, ambos en Santiago (IBID).

\subsection{Obtención del Premio Nacional de Educación 2013}

Como se desprende fácilmente de lo descrito, no es de extrañar que el Estado de Chile le haya entregado a Beatrice Ávalos el Premio Nacional de Educación 2013, aunque ella no había postulado. Sin embargo, el 2 de septiembre de 2013, el jurado compuesto por la Ministra de Educación, Carolina Schmidt; el Rector de la Universidad de Chile, Víctor Pérez; y la ganadora anterior del premio, Erika Himmel; y en representación del Consejo de Rectores: Patricio Sanhueza Vivanco, Rector de la Universidad de Playa Ancha; y Jaime Espinosa Araya, Rector de la Universidad Metropolitana de Ciencias de la Educación, le otorgaron el Premio por su aporte a la teoría sobre la profesión docente y la calidad de la enseñanza (A.A., 2013). 


\section{PENSAMIENTO EDUCATIVO DE LA DRA. BEATRICE ÁVALOS DAVIDSON}

Por lo señalado en los puntos precedentes se ha clarificado que Beatrice Ávalos se destaca como una importante educadora e investigadora en Chile y en otros países latinoamericanos e ingleses (incluidas antiguas colonias como Canadá, Papúa Nueva Guinea y Australia), pero, además, posee un pensamiento propio, expresado en múltiples publicaciones ; al analizar sus planteamientos pedagógicos se percibe su calidad investigativa y su énfasis por mejorar la calidad de la educación, preocupándose especialmente de la formación de los docentes y del ejercicio de la profesión de los mismos; para ella, el trabajo colaborativo y especialmente el apoyo de los docentes experimentados a los novatos es su preocupación central. Lo más destacado se encuentra en su preocupación por la formación de los profesores y, además, su vida ha estado marcada por aquello, pues en Chile se inició formando futuros educadores en la Universidad Católica de Chile y, en esa institución, al participar en la creación del Magíster en Educación y el PIIE, perseguía entregar una mejor educación (a través del Magíster) y, para ello, se necesitaba investigar (PIIE); luego va más allá al lograr el convenio de Doctorado en Educación con la Universidad de Gales, ya que en Chile aún no existía ese grado académico; deseaba que los académicos chilenos se perfeccionaran en un alto grado para que entregaran una mejor formación a sus alumnos. En Papúa Nueva Guinea tendrá la misma preocupación: formar mejores docentes y, por lo mismo, crea el Magíster en Educación en la universidad de ese país. Por ello, cuando regresa a su país, su preocupación será la formación inicial docente a nivel de su labor en el Ministerio de Educación y como investigadora en la Universidad de Chile. No hay que olvidar, a su vez, que en Papúa Nueva Guinea tuvo una preocupación especial por la educación de la mujer, a partir de investigaciones realizadas al respecto, patrocinadas y difundidas por el Banco Mundial.

A continuación se sintetizan los principales aspectos de algunos tópicos de su pensamiento educativo.

\subsection{Mujer, educación y desarrollo}

En los 6 años de permanencia en el país polinésico, tuvo una preocupación especial por la mujer, debido fundamentalmente a la situación de ellas: analfabetismo, bajas tasas de matrícula y asistencia escolar, tanto en escuelas como en la educación terciaria; la pobreza de las mujeres, falta de control sobre sus recursos económicos y desigualdad laboral (BROUVER et al., 1998). Por esa razón, Beatrice Ávalos aborda el tema de la mujer, tanto desde el punto de 
vista educacional como económico. Señala en un documento de trabajo de la División Económica e Investigación de Estudios de Asia Pacífico (ÁVALOS, 1994), las diferencias de género en salud, educación, empleo y participación en la toma de decisiones; realiza un análisis de las iniciativas actuales para mejorar la equidad de género y participación de las mujeres en el desarrollo y, a la vez, beneficiarse del mismo. Paralelamente, en conjunto con Ken GANNICOTT (1994), profundiza el punto anterior, indicando que internacionalmente, la educación de las mujeres ha surgido como un aspecto significativo del crecimiento económico y su desarrollo; las investigaciones en diversos países en vías de desarrollo muestra el valor de la educación de las mujeres: educar a las mujeres mejora la salud, la expectativa de vida y el PNB; por lo mismo es necesario acortar las brechas de género en la educación entre hombres y mujeres; si la brecha es alta, baja el PIB; por lo tanto, la conclusión es obvia: educar a las mujeres es la inversión más importante que se puede hacer.

Sin embargo, en el estudio empírico realizado junto a Ken Gannicott en 1993 -ella en Papúa Nueva Guinea y él en las islas Fiji, Solomon y Vanuatu- descubren que es necesario que los gobiernos mejoren las políticas públicas en los países en desarrollo para "intentar acelerar el proceso de difusión de la información sobre el valor de educar a las mujeres” (GANNICOTT; ÁVALOS, 1994, p. 1), considerando que "educar a las mujeres rinde beneficios futuros a ellas mismas, a su familia y a la sociedad en general, pero la carga de los costos a menudo corre por cuenta de los padres solamente" (IBID); de esta forma, se encuentran reacios a mandar a sus hijas a la escuela, pues ellos necesitan que sus hijas produzcan ahora y no en el futuro.

En otra publicación (ÁVALOS, 1995) concluye que el enfoque de desarrollo seguido por Papúa Nueva Guinea ha entregado bienestar, pero no ha dado igualdad, puesto que siguen existiendo brechas de género en salud, educación, empleo y toma de decisiones; por lo mismo, hace un llamado a los intelectuales, altos funcionarios públicos y políticos para eliminar la injusticia y darle a las mujeres el apoyo que requieren.

\subsection{Filosofía educacional}

Comienza a esbozar su pensamiento en su tesis doctoral desde una perspectiva filosófico-educacional y reflexiva, planteando que "en toda teoría pedagógica se encuentra siempre contenida alguna noción sobre perfección humana que le sirve de guía para la determinación de sus aplicaciones prácticas" (ÁVALOS, 1965, p. 9); luego, agrega: "Si se examina la historia del pensamiento educacional desde Platón hasta Dewey, se encuentra en ella un objetivo común: obtener una visión y comprensión de los caminos y medios por los cuales el hombre pueda alcanzar su completación como ser humano" (ÁVALOS, 1965, p. 9). 
Los fundamentos de la filosofía de la educación se encuentran allí muy presentes, pues el centro del proceso educativo es la persona del alumno y la perfectibilidad del mismo hace posible el quehacer formativo (CAICEO, 1996). Luego, recorre el pensamiento contemporáneo, citando a Kierkegaard y Nietzsche, pero especialmente a Jaspers y Marcel, existencialistas cristianos, cuyo pensamiento está presente en la autora en estudio. A continuación se centra en lo que Beatrice Ávalos denomina naturalismo y, en esa línea, analiza el pensamiento de Marx y Dewey. Sobre el primero señala que su humanismo ha confundido al ser humano solo como una fuerza productiva y "al sumergir al individuo en lo colectivo, no lo ha salvado, sino que, por el contrario, lo ha funcionalizado $\mathrm{y}$, virtualmente, ha suprimido su existencia como persona" (ÁVALOS, 1965, pp. 82-83). En relación al segundo, acota que "ha comprometido peligrosamente la existencia individual, la creatividad y la libertad, al dejar en manos de las fuerzas sociales, que están operando en el medio ambiente del hombre, toda su formación, y ha descuidado proveer lo necesario para cualquier realidad particular e individual que trascienda esas fuerzas" (ÁVALOS, 1965, p. 83). Sin embargo, reconoce que ambos humanismos naturalistas han puesto el tema de la educación en un contexto de realidad social, cultural y de desarrollo científico-tecnológico que es necesario tener presente: es encontrarse con el educando real y el mundo real y no con una abstracción del mismo y de su realidad. Frente a esta situación, propone que hay que descubrir al hombre nuevo en una nueva comunidad; por lo mismo,

[...] en oposición al hombre desarraigado tenemos que educar a una persona vinculada firmemente, con lazos conscientes y duraderos a los objetos naturales de sus necesidades: personas, ideas, hogar [...]. Con el fin de contrarrestar el impacto del hombre-masa, conformista, debemos hacer uso de fuerzas sociales (como instituciones educacionales y acción de grupo) y por medio de ellas, debemos formar una persona capaz de reflexionar, actuar libremente y con responsabilidad en función de decisiones personales [...]. Finalmente, para superar la soledad que persigue al hombre moderno es nuestra tarea ayudarlo a desarrollar y a recuperar su capacidad de amar [...] (ÁVALOS, 1965, p. 199).

Como se puede desprenderse de sus palabras, ella estaba imbuida del concepto cristiano de hombre al considerarlo persona y de valores propios de ese pensamiento, como el de participación en comunidad, enfatizar el desarrollo personal del educando, el amor al otro, la solidaridad. Sin embargo, tales valores podrían considerarse universales y no exclusivos del cristianismo y así lo ha puesto en práctica a lo largo de su vida académica y personal la doctora Ávalos. 


\subsection{Investigaciones empíricas: Formación docente, trabajo colaborativo, formación inicial}

En las publicaciones derivadas de sus investigaciones posteriores se refleja un cambio en su labor profesional: sin desconocer el valor de la antropología filosófica, como iluminadora del quehacer educativo, su trabajo lo orienta más a la investigación empírica, recogiendo información importante para la toma de decisiones a nivel de políticas públicas por parte de autoridades gubernamentales; de esta manera, ha quedado muy claro su calidad investigativa y su preocupación por mejorar la calidad de la educación a partir de la realidad; ha tomado conciencia que es necesario centrarse especialmente en la formación de los docentes y en el ejercicio de la profesión de los mismos; para ella, el trabajo colaborativo y, especialmente el apoyo de los docentes experimentados, a los novatos es su preocupación central.

Por lo mismo, en relación al desarrollo de la profesión docente señala:

Los profesores que comienzan a enseñar, durante su primer año de trabajo, debieran tener profesores mentores, que es algo distinto a lo que ocurre en las prácticas que se realizan en la Formación Inicial. Son muchos los beneficios que se obtienen con un sistema de inducción y mentorías, y así lo evidencian distintos estudios, pues puede ser una ayuda para el joven profesor cuando enfrenta situaciones complejas, evitando el abandono de la profesión, e igualmente permite reducir la movilidad del profesor entre un liceo y otro sin anclarse a un centro escolar. En síntesis, estos sistemas mejoran la práctica docente de los profesores, los apoyos elevan el sentido de autoeficacia del profesor, y favorecen la construcción y reconstrucción de la identidad profesional (ÁVALOS, In VILLA, 2015, s/p).

Lo relevante de su planteamiento es que tales ideas se incluyeron en la ley $\mathrm{N}^{\circ} 20.903$ que se publicó en el Diario Oficial al año siguiente (BIBLIOTECA DEL CONGRESO NACIONAL, 2016). Ella las estaba trabajando en el Proyecto Conicyt , que lideraba en el 2015 Aprendizaje profesional docente colaborativo en contextos escolares: Oportunidades y limitaciones. Sin embargo, la relación entre la formación inicial del profesor y su desarrollo en el ejercicio de su profesión, lo venía trabajando desde años antes la Dra. Ávalos con equipos del CIAE de la Universidad de Chile; en efecto, en un artículo con algunos de sus colaboradores, concluye:

Si entendemos la profesionalización como resultado de una temporalización en la adquisición de competencias profesionales, no habría cabida para la convergencia de expertos y novatos en un proceso común. Sin embargo, si aceptamos el hecho que la concientización no obedece a ritmos preestablecidos ni a formatos consecutivos en la formación docente, veremos claramente que novatos y expertos pueden formar comunidad profesional en busca de sentidos, donde la dialéctica abra espacios a la articulación teoríapráctica. En efecto, los profesores novatos o en formación suelen estar más cerca de los enunciados teóricos. Del mismo modo, los profesores expertos 
están más cerca de los enunciados prácticos. Generar el espacio dialógico que permita confrontar ambos enunciados o ambas perspectivas, resultaría en un evidente enriquecimiento de las partes (NÚÑ̃Z et al., 2012, s/p).

Sin embargo, su preocupación por la formación inicial es de larga data y en un estudio publicado de una investigación Conicyt (ÁVALOS, 2016, s/p), señala:

La evolución y características de la formación inicial docente durante los últimos quince años ha sido objeto de diversos estudios centrados en el análisis de las políticas de formación docente (COX et al., 2010, pp. 205-245), en su efectividad para producir conocimiento pedagógico general y de contenido matemático (ÁVALOS \& MATUS, 2010, p. 28) y en la relación entre calidad de las instituciones en que se albergan los programas de formación docente y el nivel de conocimiento de los futuros profesores (PEDRAJA-REJAS et al., 2012, p. 18).

Considerando la experticia de la Profesora Ávalos en el tema de la Formación Inicial Docente, la Unesco le encomendó un estudio a nivel de América Latina y El Caribe respecto al tema, logrando abarcar Argentina, Brasil, Chile, Colombia, Guatemala, México, Perú, y el Caribe Anglófono. En 2011 elabora un informe, en el cual hace notar la importancia del tema:

[...] en relación a los resultados de aprendizaje insatisfactorios de los alumnos de la Región en las pruebas nacionales estandarizadas y en las pruebas internacionales. Se indican las siguientes áreas críticas que emergen del estudio de la situación: diferencias en la dependencia institucional de la formación docente (escuelas normales, institutos superiores, universidades), la calidad de los programas de formación, las condiciones de regulación, las diferencias en el clima institucional entre normales y universidades y la tensión entre formación 'universalista' y formación diferenciada para los grupos sociales (ÁVALOS, 2011, p. 3).

En el mismo informe describe políticas emergentes en vistas a mejorar la futura formación docente, tema mencionado precedentemente.

\subsection{Reflexiones y aportes a la educación en el Chile actual}

Para Burrows (2014, p. 3) lo que ha hecho Beatrice Ávalos en educación en Chile "ha sido un aporte extraordinario, para mí, principalmente en la formación de educadores con una visión de la educación centrada en la persona”. A su vez, agrega que “[...] siendo director de la Sede Villarrica de la Universidad Católica de Chile, la invité para que diera una clase magistral sobre el desarrollo de competencias en el educador de este siglo" (BURROWS, 2014, p. 3).

En relación a la coyuntura en que se ha visto envuelta la educación en los últimos 45 años en el país, Beatrice Ávalos hace la siguiente reflexión: 
La educación efectivamente tiene un rol de cambio, pero a su vez sufre el embate de la sociedad y el potencial de la educación muchas veces es coartado por fuerzas sociales y políticas que la obstruyen. Ese es el tremendo problema que enfrentamos no solo en Chile: la instrumentalización de la educación para producir el tipo de persona necesaria para el desarrollo económico y para la competitividad. Esa contradicción es la que enfrentan actualmente las políticas en Educación. Por un lado deben entender el rol de cambio de la Educación; pero por otro, estar empujados por esta noción de Educación al servicio del desarrollo económico y del mercado. Y es en esa lógica en la que se inserta nuestro sistema educacional competitivo, con el financiamiento de los vouchers. De hecho, los alumnos de la educación pública municipalizada se mueven a la particular subvencionada (ÁVALOS, 2013, s/p).

En cuanto al proceso de desmunicipalización de la educación chilena, opinaba en el 2014:

La mayor parte de los municipios no tienen las capacidades para manejar bien la educación, los liceos y las escuelas bajo su dirección. Eso no quiere decir que haya algunas que lo hacen bastante bien. Lo complicado yo creo es qué se reemplaza por la educación municipal. El financiamiento viene del Estado, pero el tema es encontrar formas descentralizadas, porque tampoco se puede centralizar todo en el edificio del Ministerio de Educación; por lo tanto, hay que buscar formas descentralizadas de administrar la educación pública chilena. Yo creo que eso es parte de lo que se está discutiendo y se están armando y se está trabajando en propuestas ahora en el Ministerio de Educación. Ha habido propuestas en los últimos años de Agencias Locales de Educación, yo creo que esa parece ser la más razonable, pero el tema es qué quiere decir lo local, si es la comuna, la provincia, la región, entonces yo creo que todo eso está en discusión (ÁVALOS, 2014b, p. 7).

El 24 de noviembre de 2017 se publica la Ley № 21.040 sobre la Nueva Educación Pública, la cual establece que existirán 71 Agencias Locales de Educación en todo el país. 4 de ellas comenzarán a funcionar en forma piloto en el 2018 y paulatinamente se irán integrando las demás, previa evaluación del proceso. De esta forma, las municipalidades del país irán devolviendo los establecimientos educacionales al estado. Se concretaron los deseos de la Premio Nacional.

El 1988 se creó la prueba estandarizada SIMCE (Sistema de Medición de la Calidad de la Educación) que inicialmente se aplicaba a todos los cuartos y octavos de la educación básica en las asignaturas de castellano y matemáticas (CAICEO, 2015); sin embargo, en el primer gobierno de Sebastián Piñera (2010-2014) se comenzó a aplicar en segundo y sexto de enseñanza básica y en segundo y tercero de enseñanza media y en más asignaturas (ciencias sociales, ciencias naturales e inglés), exagerando las mediciones; de esta forma, los establecimientos educacionales se dedicaban a preparar a sus estudiantes para dar la prueba SIMCE y no para educar de acuerdo a los planes y programas vigentes. Esto llevó a que nuestro 
Premio Nacional encabezara un movimiento, titulado Alto al SIMCE en el 2013. Lo cual tuvo sus efectos en los años siguientes disminuyendo la cantidad de pruebas y niveles y sus resultados no se publican en rankings públicos sino que se entregan a los establecimientos educacionales para que revisen sus procesos pedagógicos y mejoren los aspectos insuficientes que entrega la prueba. Al respecto, Beatrice Ávalos recuerda:

Un joven investigador, quien es uno de mis ayudantes, Felipe Acuña, se acercó y me pidió que firmara la carta, dándome la posibilidad de cambiar lo que quisiera. En la primera parte estuve de acuerdo, pero no me gustó una sección que terminaba con 'rechazamos'. La cambié y relativicé ese término por 'señalamos nuestra oposición a'. Esta carta se origina en un grupo de investigadores jóvenes de los cuales hay algunos que han levantado el movimiento 'Alto al SIMCE' en la Facultad de Ciencias Sociales de la Universidad de Chile (ÁVALOS, 2013, s/p).

La importante educadora chilena no está segura de que la gratuidad universitaria sea posible para todos y que ella vaya a mejorar la calidad de la educación. Esta afirmación la hizo en el 2013; en enero de 2018 el Congreso Nacional aprobó una ley al respecto con gratuidad universal; hasta el momento se aplica al $60 \%$ de los alumnos con menores ingresos, pero para llegar a cubrir el $100 \%$ pasará mucho tiempo. Textualmente señala:

Creo que la educación superior gratuita y de calidad va a ser difícil, dado lo complejo que es financiar las universidades, salvo que el Estado ponga un montón de plata. Lo veo a partir del propio Centro de Investigación Avanzada en Educación, que recibe financiamiento básico de Conicyt, pero que necesita más, por lo que termina vendiendo servicios. Y estos se pueden vender a los buenos y a los malos. Pero esto en sí no es lucro, es dinero que sirve para mantener a los investigadores, para tener áreas de investigaciones que no se financian (ÁVALOS, 2013, s/p).

Con la Iglesia Católica ha colaborado cuando le han solicitado sus servicios; así, por ejemplo, en la Vicaría para la Educación del Arzobispado de Santiago dirigió el denominado Plan Maestro, el cual fue presentado al gobierno de Michelle Bachelet por ella y el Vicario para la Educación, P. Tomás Scherz. En la Universidad Católica de Chile se ha integrado al Doctorado en Educación con clases y supervisión de tesis (ÁVALOS, 2017).

\section{CONCLUSIONES}

Los objetivos planteados inicialmente se han cumplido a cabalidad, puesto que se ha reconstruido la vida de esta importante educadora chilena, tanto en Chile como en el extranjero y se han descrito sus actividades académicas, tanto como docente como investigadora de la 
educación en diversos países, destacando su autoexilio por 20 años en Gran Bretaña, Canadá y Papúa Nueva Guinea. A su vez, se ha realizado un esbozo de su pensamiento educativo, partiendo por la filosofía de la educación y continuando con el tema que más le apasiona, la formación inicial docente, sin descuidar su incursión en el tema de la educación de la mujer y lo que implica para el desarrollo de los pueblos que ellas también tengan una educación de calidad. Es tan extenso su trabajo investigativo en pro de mejorar las políticas públicas en la formación de profesores que este trabajo se alargaría demasiado; su preocupación por mejorar o cambiar las políticas públicas con evidencia empírica se extiende a países latinoamericanos, del Caribe y de la Oceanía, centrándose en los últimos 25 años en su país, Chile.

$\mathrm{Su}$ accionar revela que cuando una mujer desarrolla todas sus potencialidades no solo puede igualar a los hombres, sino que incluso, superarlos. Entre sus preocupaciones y, se demuestra en este artículo, ha estado el desarrollo personal y laboral de la mujer de menores ingresos y ha luchado porque desaparezca la brecha educativa y salarial entre hombres y mujeres, problema muy importante en el mundo de hoy, especialmente en los países que aún no han alcanzado el desarrollo.

\section{REFERENCIAS}

A.A. Beatrice Ávalos Davidson obtiene Premio Nacional de Ciencias de la Educación 2013, 2013. Disponível em: https://www.emol.com/noticias/nacional/2013/09/02/617738/beatriceavalos-davidson-obtiene-premio-nacional-de-ciencias-de-la-educacion-2013.html. Acesso em 21 de mai. 2019.

ÁVALOS, Beatrice. New men for new times. New York: Sheed and Ward, 1962.

ÁVALOS, Beatrice. Nuevos hombres para nuevos tiempos. Santiago de Chile: Editorial Salesiana, 1965.

ÁVALOS, Beatrice. Women and development in papua new guinea. Canberra: Economic Division Working Papers, Research School of Pacific and Asian Studies, 1994.

ÁVALOS, Beatrice. Women and Development. Pacific Economic Bulletin 10 (1): 73-83, 1995. Disponível em: https://www.google.com/search?client=firefox-b$\mathrm{d} \& \mathrm{q}=$ Beatrice + Avalos $\% 2 \mathrm{C}+\% 22 \mathrm{Women}+\mathrm{and}+$ Development $\% 22 \% 2 \mathrm{C}+\mathrm{Pacific}+$ Economic $+\mathrm{B}$ ulletin $+10 \% 281 \% 29+\% 281995 \% 29 \% 3 \mathrm{~A}+73-83$. Acesso em 21 mai 2019.

ÁVALOS, Beatrice; MATUS, Claudia. La formación inicial docente en Chile desde una óptica internacional: informe nacional del estudio internacional LEA TEDS-M. Santiago de Chile: Ministerio de Educación, 2010.

ÁVALOS, Beatrice. Formación Inicial Docente: Proyecto Estratégico Regional sobre Docentes UNESCO-OREALC/CEPPE. Santiago de Chile: UNESCO, 2011. 
ÁVALOS, Beatrice. Entrevista. Protagonista del artículo, realizada por Boris Bezama en Santiago de Chile y publicada en CIPER Chile el 3 de octubre de 2013 con el título: La devastadora noticia que opacó la celebración de la Premio Nacional de Educación 2013, 2013. Disponível em: https://www.ciperchile.cl/2013/10/03/la-devastadora-noticia-que-opaco-lacelebracion-de-la-premio-nacional-de-educacion-2013/. Acesso em 21 mai 2014.

ÁVALOS, Beatrice en SALINAS, Juan Luis Las Lecciones de Beatrice Ávalos, Premio Nacional de Educación 2013. Revista Ya de El Mercurio, Santiago de Chile (martes 14 de enero), pp. 43-45, 2014a.

ÁVALOS, Beatrice. Entrevista. Protagonista del artículo, realizada por Carmen Gloria Carrasco Zanocco en Santiago de Chile el 15 de junio, 2014b.

ÁVALOS, Beatrice. Curriculum Vitae. Protagonista del artículo, entregado por la misma doctora al autor de este texto en Santiago de Chile, 2015a.

ÁVALOS, Beatrice. Curriculum Vitae Descriptivo. Protagonista del artículo, entregado por la misma doctora al autor de este texto en Santiago de Chile, 2015b.

ÁVALOS, Beatrice. La formación inicial docente en Chile: tensiones entre políticas de apoyo y control. Estudios Pedagógicos, Valdivia, v. 40, n. Especial, p. 11-28, 2016. Disponível em: https://scielo.conicyt.cl/scielo.php?script=sci_arttext\&pid=S071807052014000200002\&lng=es\&nrm=iso. Acesso em 15 abr. 2019.

ÁVALOS, Beatrice. Entrevista. Protagonista del artículo, realizada por el autor de este texto en Santiago de Chile el 10 de octubre, 2017.

BIBLIOTECA DEL CONGRESO NACIONAL. Período 1925-1973: Profundización y crisis de la democracia, 1925-1973. Disponível em:

https://www.bcn.cl/historiapolitica/hitos_periodo/detalle_periodo.html?per=1925-1973.

Acesso em 9 mai. 2019.

BIBLIOTECA DEL CONGRESO NACIONAL. Ley $N^{\circ}$ 20.903: Crea el Sistema de Desarrollo Profesional Docente y Modifica otras Normas. Santiago de Chile: Diario Oficial, $1^{\circ}$ de abril de 2016. Disponível em: https://www.leychile.cl/Navegar?idNorma=1087343. Acesso em 21 mai. 2019.

BIBLIOTECA DEL CONGRESO NACIONAL. Ley $N^{\circ}$ 21.040: Crea el Sistema de Educación Pública. Santiago de Chile: Diario Oficial, 24 de noviembre de 2017. Disponível em: https://www.bcn.cl/leychile/navegar?idNorma=1111237. Acesso em 21 mai. 2019.

BROUVER, Elizabeth; HARRIS, BRUCE \& TANAKA, Sonomi (Editors). Gender Analysis in Papua New Guinea. Washington: The World Bank, 1998.

BURROWS, Fernando. Entrevista. Discípulo de la Protagonista, realizada por Carmen Gloria Carrasco Zanocco en Santiago de Chile el 10 de julio de 2014.

CAICEO, Jaime. Proyecto Educativo Cristiano: fundamentos antropológicos, educativos y sociales. Santiago de Chile: Ediciones Facultad de Educación de la Universidad Católica Blas Cañas, 1996. 
CAICEO, Jaime. P. Patricio Cariola; Premio Nacional en Educación y su Paso por la Fide. Revista de Pedagogía 472: 32-40, 2012a.

CAICEO, Jaime. Pensamiento y Acción Educativos en Chile a partir de los Premios Nacionales de Ciencias de la Educación, Tomo I. Santiago de Chile: Ediciones Universidad de Los Lagos, 2012b.

CAICEO, Jaime. Los Sistemas Estandarizados de Evaluación en Chile: participación de Mario Letyton Soto y Erika Himmel König. Historia de la Educación. Revista Interuniversitaria, v. 34, pp. 357-371, 2015. Disponível em: https://revistas.usal.es/index.php/0212-0267/article/view/hedu201534357371. Acesso em 13 dez. 2020.

CAICEO, Jaime. La Pedagogía de Dewey en Chile: su presencia, a través de sus discípulos, durante el siglo XX. Santiago de Chile: Ediciones Departamento de Contabilidad y Auditoría y Departamento de Educación de la Universidad de Santiago de Chile, 2016.

CAICEO, Jaime. El Porqué del Desarrollo Insuficiente de la Filosofía de las Ciencias Sociales. El Futuro del Pasado, v. 9, pp. 393-418, 2018. Disponível em: https://www.elfuturodelpasado.com/ojs/index.php/FdP/article/view/318/302. Aesso em 13 dez. 2020;

COX, Cristián; MECKES, Lorena; BASCOPÉ, Martín. La institucionalidad formadora de profesores en Chile en la década del 2000: velocidad del mercado y parsimonia de las políticas. Pensamiento Educativo, vols. 46-47, 2010. pp. 205-245. Disponível em: https://pensamientoeducativo.uc.cl/files/journals/2/articles/468/public/468-1034-1-PB.pdf. Acesso em 10 abr. 2019.

GANNICOTT, Ken; ÁVALOS, Beatrice. Women's Education and Economic Development in Melanesia (Canberra: National Centre for Development Studies, Research School of Pacific and Asian Studies, Australian National University, 1994.

GONZÁLEZ, Luis Eduardo. Entrevista. Discípulo de la Protagonista, Doctor en Educación, Académico del PIIE, realizada por Carmen Gloria Carrasco Zanocco en Santiago de Chile el 20 de junio de 2014.

NÚÑEZ, Mauricio Alejandro; ARÉVALO, Ana; ÁVALOS, Beatrice. Profesionalización docente: ¿Es posible un camino de convergencia para expertos y novatos? Revista Electrónica de Investigación Educativa - REDIE, v. 14, n² 2, p. 10-24, jan. 2012. Disponível em: http://www.scielo.org.mx/scielo.php?script=sci_arttext\&pid=S160740412012000200002\&lng=es\&nrm=iso. Acesso em 7 abr. 2019.

PEDRAJA-REJAS, Liliana; ARANEDA-GUIRRIMAN, Carmen; RODRÍGUEZ-PONCE, Emilio; RODRÍGUEZ-PONCE, Juan J. Calidad en la formación inicial docente: evidencia empírica de las universidades chilenas. Formación Universitaria, v. 5, n.4, p. 15-26, 2012. Disponível em: https://scielo.conicyt.cl/pdf/formuniv/v5n4/art03.pdf. Acesso em 21 mai. 2019.

VILLA, Cristian. Premio Nacional de Educación 2013, Beatrice Ávalos, destaca beneficios de sistema de inducción a profesores en la Universidad Bío-Bío. Noticias UBB, 24 de noviembre de 2015. Disponível em: http://noticias.ubiobio.cl/2015/11/24/premio-nacional-de- 
educacion-2013-beatrice-avalos-destaca-beneficios-de-sistema-de-induccion-a-profesores-enla-ubb/. Acesso em 7 de abr. 2019.

\section{SOBRE O AUTOR}

Jaime Caiceo Escudero é doutor em Ciências da Educação pela Pontificia Universidad Catolica Argentina Santa Maria, de Buenos Aires. É docente do Departamento de Educação da Universidade de Santiago de Chile.

Email: jcaiceo@hotmail.com

ORCID: https://orcid.org/0000-0002-2808-140X

Recebido em 23 de julho de 2020. Aprovado em 27 de novembro de 2020. Publicado em 23 de dezembro de 2020 